\title{
Acknowledgement to Reviewers of Children in 2017
}

\section{Children Editorial Office}

MDPI AG, St. Alban-Anlage 66, 4052 Basel, Switzerland

Published: 23 January 2018

Peer review is an essential part in the publication process, ensuring that Children maintains high quality standards for its published papers. In 2017, a total of 115 papers were published in the journal. Thanks to the cooperation of our reviewers, the median time to first decision was 28 days and the median time to publication was 57 days. The editors would like to express their sincere gratitude to the following reviewers for their time and dedication in 2017:

\begin{tabular}{ll} 
Abramo, Thomas J. & Bremer, Andrew A. \\
Agarwal, Amit & Brennan, Patricia \\
Alaofe, Halimatou & Breuner, Cora Collette \\
Allegaert, Karel & Brierley, Daniel \\
Allen, Taryn & Brilli, Richard \\
Amini, Rose Marie & Brockbank, Justin \\
Arca, Marcello & Brown, Melanie \\
Ardigò, Luca & Brown, Samantha \\
Arnbjörnsson, Einar & Bruyneel, Luk \\
Asakura, Keiko & Buchowski, Mac \\
August, Keith J. & Butler, Marilyn W. \\
Ayán Pérez, Carlos & Cabanillas, Beatriz \\
Baker, Emma & Cady, Rhonda G. \\
Baker, Mark Douglas & Caicedo, Carmen \\
Barbi, Egidio A. & Camenga, Deepa R. \\
Barnes, Gregory & Canbay, Ali \\
Barone, Daniel A. & Canu, Will \\
Barton, Karen & Carney, Paul R. \\
Beck, Suzanne E. & Carr, Robert A. \\
Becker, David & Carter, Brian S. \\
Bender, Hans-Ulrich & Casanovas, Carmen \\
Berens, Pamela D. & Cemeroglu, Ayse Pinar \\
Berezin, Eitan & Chadi, Nicholas \\
Berger, Rolf & Cheung, Po-Yin \\
Bergstraesser, Eva & Chhatwal, Jugesh \\
Beslow, Lauren A. & Chilaka, Cynthia Adaku \\
Bhandari, Vineet & Chirica, Mircea \\
Bhat, Sushanth & Chung, Dai H. \\
Biank, Vincent F. & Ciccone, Marco Matteo \\
Bisulli, Francesca & Ciciolla, Lucia \\
Blackall, Douglas P. & Clemente, Maria Grazia \\
Blankestijn, Mark & Clinch, Jacqui \\
Borden, Lynne & Cohen, Arieh Sierra \\
Boshuizen, Henny P.A. & Cohen, Eyal \\
Boss, Renee & Constance, Jonathan E. \\
Bravaccio, Carmela & Contini, Sandro \\
& \\
\hline
\end{tabular}


Cotton, Robin T.

Cristea, Ioana

Crowe, Suzanne

Cruz, Maria Leticia

Culbert, Timothy P.

Cunningham, Melody

Daikeler, Thomas

Dampier, Carlton

Davison, Kirsten

De La Vega, Rocio

De Schweinitz, Peter Alan

DeJesus, Jasmine

Dhanekula, Raja Koteswar

Di Martino, Giuseppe

Dick, Bruce

Dimopoulos, Konstantinos

Dore-Stites, Dawn Jeanette

Fenaughty, Andrea M.

Ferrara, Christine

Fink, Ericka L.

Fitzpatrick, Emer

Flaxman, Abraham

Fleury, Marie-Josée

Flexeder, Claudia

Francois, Katrien

Friedland-Little, Joshua M.

Friesen, Myron D.

Fucho, Raquel

Furman, Lydia

Gardner, Renee

Gari, Merce

Geisler, Corinna

Geyer, James D.

Gholami, Maryam

Giardini, Alessandro

Gien, Jason

Godse, Alok

Goldman-Mellor, Sidra

Golianu, Brenda

Gomez, Camilo

Gordon, John

Graham, H. Kerr

Greenfield, John

Greenough, Anne

Gruber, Kenneth J.

Guinhouya, Benjamin C.

Hall, David

Hamati, Aline

Han, Seung Jin

Hao, Lei

Harding, Celia

Harris, William
Hassan, Sherif T.S.

Hauer, Julie Marie

Hazell, Philip

Hepping, Nico

Herbert, Anthony

Hermos, Christina

Himmelmann, Kate

Hjern, Anders

Hohwu, Lena

Holmes, Geoffrey

Hosie, Annmarie

Hudson, Darrell L.

Hughes, Jean R.

Humphrey, Lisa

Humpl, Tilman

Iceland, John

Imdad, Aamer

Iwamoto, Shotaro

Jaaniste, Tiina

Jain, Amish

Japaridze, Natia

Jastrowski, Mano Kristen E.

Jegaskanda, Sinthujan

Jena, Prasant

Jetelina, Katelyn Kassarjian

Job, Kathleen

Jones, Chrissie

Kaiser, Pamela

Kang, Bum-Yong

Kaplan, Edward Lawrence

Katwa, Umakanth

Kavanagh, Katherine F.

Kavanagh, Patricia

Keenan, Mickey

Kemper, Kathi

Kim, Harris Hyun-soo

Kini, Usha

Kirsch, Roxanne

Kocken, Paul L.

Kohen, Daniel P.

Kolen, Angela M.

Koot, Bart G.P.

Kotagal, Suresh

Kovesi, Tom

Lacy, Katie

Lakshminrusimha, Satyan

Lane, Shelly

LaRovere, Kerri L.

Lavine, Joel

Lee Hon, Wah

Lightman, Susan

Lim, Crystal 
Litt, Johnathan

Long, $\mathrm{Xi}$

Lopes, Luis

Lopez, Nanette

Lucendo, Alfredo

Lucey, Alice

Ma, Zheng Feei

Maclellan, Reid A.

Mahieu, Ludo

Malby Schoos, Ann-Marie

Mandrell, Belinda

Mars, Maurice

Martin, Stephanie

Maslin, Kate

Massotte, Dominique

Mattsson, Janet

Mayer, Anton

McDonnell, Andrew

McIntosh, Ian

McIntosh, E. David G.

McKenna, Malachi J.

McLoney, Eric D.

Mearelli, Filippo

Metzger, Monika L.

Meyer, Walter J.

Mignon, Sylvia

Misra, Sanghamitra M.

Moise, Imelda K.

Monk, Ellis P.

Morrison, Wynne E.

Muñoz-López, Francisco

Munshi, Aditya

Musher-Eizenman, Dara R.

Mutiga, Samuel K.

Naim, Mitre

Nakai, Kunihiko

Natale, Vincenzo

Nazare, Barbara

Newby, Ruth

Newmark, Sanford

Neymotin, Florence

Nobmann, Elizabeth D.

Noel, Melanie

Noonan, Vikki

Noritz, Garey

Nuss, Henry J.

O'Rourke, Margaret

Okhuysen-Cawley, Regina

O'Malley, Lucy

Oostenbrink, Rianne

Ortiz, Robin

O'Shea, Joyce
Osier, Nicole

Owili, Patrick

Pacifico, Lucia

Pallikkuth, Suresh

Palm, Øyvind

Paradies, Yin

Parkes, Alison

Patman, Shane

Pavone, Martino

Payne, Peter

Pease, Anna

Peltz, Alon

Pendergrast, Robert

Peraica, Maja

Pérez-Rodrigo, Carmen

Pezzella, Angelo Thomas

Pfeiffer, Beth

Phillips, Farya

Pielech, Melissa

Pinheiro, Joaquim

Pinquart, Martin

Pinto Guedes, Dartagnan

Pittenger, Jaime

Pokorska-Śpiewak, Maria

Prabhakaran, Sreekala

Pratt, Iain S.

Principe, Maria Ilaria Del

Raffone, Antonino

Ramadan, Hassan H.

Rambaud, Jerome

Ramtekkar, Ujjwal

Rathinasabapathy, Anandharajan

Rempel, John K.

Robson, Shannon M.

Rollins, Nancy

Romo-Palafox, Maria J.

Rosen, Larry

Rossignol, Daniel

Rössler, Jochen

Routledge, Michael

Rüegger, Christoph

Ruskin, Danielle

Russell, Alexandra C.

Rusy, Lynn M.

Sanchez-Solis, Manuel

Sanders, Lee

Sandler, Irwin

Sandoval, Claudio

Sankarasubramanian, Vishwanath

Santoro, Nicola

Sawni, Anju

Sayón-Orea, Carmen 
Schaefer, Michael

Schafer, Ellen J.

Schechter, Neil

Schlarb, Angelika

Schlueer, Barbara

Schulze-Neick, Ingram

Schwantes, Scott

Sequeira, Sonia

Serai, Suraj D.

Sherafat-Kazemzadeh, Roya

Sherwin, Catherine M.T.

Shindou, Hideo

Shoji, Kensuke

Shonkoff, Eleanor

Shroff, Manohar

Shub, Mitchell D.

Sibinga, Erica

Sigmund, Erik

Simon, Patrick

Simons, Laura

Singh, Chanpreet

Skoro-Sajer, Nika

Slaughter, James

Sluss, Dorothy

Solah, Vicky A.

Soma, Caelan

South, Andrew

Spetea, Mariana

Stage, Virginia

Stanley, Maria A.

Sterrett, Emma M.

Stores, Gregory

Stormon, Michael

Sulman, Cecille G.

Sweeney, Brooke

Tang, Minghua

Tarini, Beth Anne

Taylor, C. Barr

Teicher, Carrie Lee
Telford, Rohan M.

Thompson, Maxine S.

Thomsen, Isaac

Thomson, Joanna

Tibboel, Dick

Tiniakou, Dina

Tokuyama, Shogo

Toossi, Saied

Tran, Susan T.

Tulloh, Robert

Turner, Elizabeth

Tutor, James D.

Vahlkvist, Signe

Vajro, Pietro

Van Baar, Anneloes

Van De Wetering, Marianne Desiree

Van Der Veek, Shelley

Veatch, Olivia J.

Viala, Jerome

Volkert, Valerie

Wallace, Dustin

Weaver, Meaghann S.

Weiner, Shira Schecter

Weiss, Margaret

Weiss, Shelly

Weydert, Joy

White, Mary L.

Wilsey, Michael

Witkamp, Frederika Erica

Wong, Judith Ju-Ming

Wright, Nicola

$\mathrm{Wu}$, Tai-wei

$\mathrm{Wu}$, Feitong

$\mathrm{Xu}$, Feng-Lian

Yunus, Fakir

Zadeh, Sima

Zandieh, Stephanie O.

Zapolski, Tamika

Zurynski, Yvonne

(C) 2018 by the authors. Licensee MDPI, Basel, Switzerland. This article is an open access article distributed under the terms and conditions of the Creative Commons Attribution (CC BY) license (http://creativecommons.org/licenses/by/4.0/). 